\title{
Overview of prognostic factors in adult gliomas
}

\author{
Akanksha Sharma ${ }^{1}$, Jerome J. Graber ${ }^{2}$ \\ ${ }^{1}$ Hospice and Palliative Medicine, University of Washington Medical Center, Seattle, WA, USA; ${ }^{2}$ Department of Neurology and Neurosurgery, \\ University of Washington Alvord Brain Tumor Center, Seattle, WA, USA \\ Contributions: (I) Conception and design: All authors; (II) Administrative support: None; (III) Provision of study materials or patients: None; (IV) \\ Collection and assembly of data: All authors; (V) Data analysis and interpretation: None; (VI) Manuscript writing: All authors; (VII) Final approval of \\ manuscript: All authors. \\ Correspondence to: Akanksha Sharma, MD. Department of Palliative Medicine, University of Washington Medical Center, 325 9th Avenue, Seattle, \\ WA 98104, USA. Email: ekakanksha@gmail.com.
}

\begin{abstract}
Gliomas represent the majority of malignant central nervous system tumors, with the most aggressive subtype, glioblastoma, accounting for almost $57 \%$ of this entity. Type of glioma and its incidence can vary depending on the age of presentation. In turn, outcomes can vary significantly based on the actual type of glioma (histologically and molecularly) and age of the patient, as well as various tumor specific factors such as size, location, comorbidities, etc. In the last decade we have been able to identify key molecular features that have provided us with greater insight into the behavior of these tumors, but the spectrum of treatment options remains limited. In addition, ultimate causes of death in patients with gliomas are variable and stochastic in nature. Given these complicated factors, prognostication for gliomas remains extremely difficult. This review aims to discuss prognostication in low grade versus high grade gliomas, variability in treatment of these tumors, clinical features of poor prognosis, and differences in prognostic understanding between patients, caregivers, and providers. We will also make some general recommendations where appropriate on how to approach this subject from a palliative care perspective.
\end{abstract}

Keywords: Brain tumors; glioma; glioblastoma; molecular tumors

Submitted Mar 14, 2020. Accepted for publication Jul 23, 2020.

doi: 10.21037/apm-20-640

View this article at: http://dx.doi.org/10.21037/apm-20-640

\section{Overview of gliomas}

The general term glioma encompasses astrocytomas, oligodendrogliomas, ependymomas, and mixed neuronalglial tumors. Gliomas are generally graded from World Health Organization (WHO) stage I through IV depending on their level of differentiation, with grade I gliomas being the most differentiated and the least malignant. Glioblastomas are grade IV gliomas-they are the most anaplastic, the least differentiated and are considered the most malignant of the tumors. While the staging of the tumor can be dependent on the mitotic activity, necrosis, and vascular proliferation that is noted pathologically, the characterization of the glioma into the various subtypes has in recent years been a marriage of histological and molecular features. The most recent WHO classification of gliomas was updated in 2016 and included some important updates around molecular integration which has allowed for increased clarity and objectivity in many cases (Figure 1) (1). For example, a glioma that appears to have oligodendroglial features on histology can only be officially named an oligodendroglioma if it has appropriate chromosomal deletions in chromosome 1 and 19 (codeletion 1p19q). Specific examples of this as it may relate into prognosis and survival are discussed at different time points throughout this review.

Causes of death in patients with gliomas (and metastatic brain tumors) are actually highly variable, and common to many neurologic diseases. Herniation leading to neurologic loss of cardiac and respiratory function is a common final event, but was only found in $60 \%$ of patients in one autopsy study (2). In the same large series, $20 \%$ of patients had an 


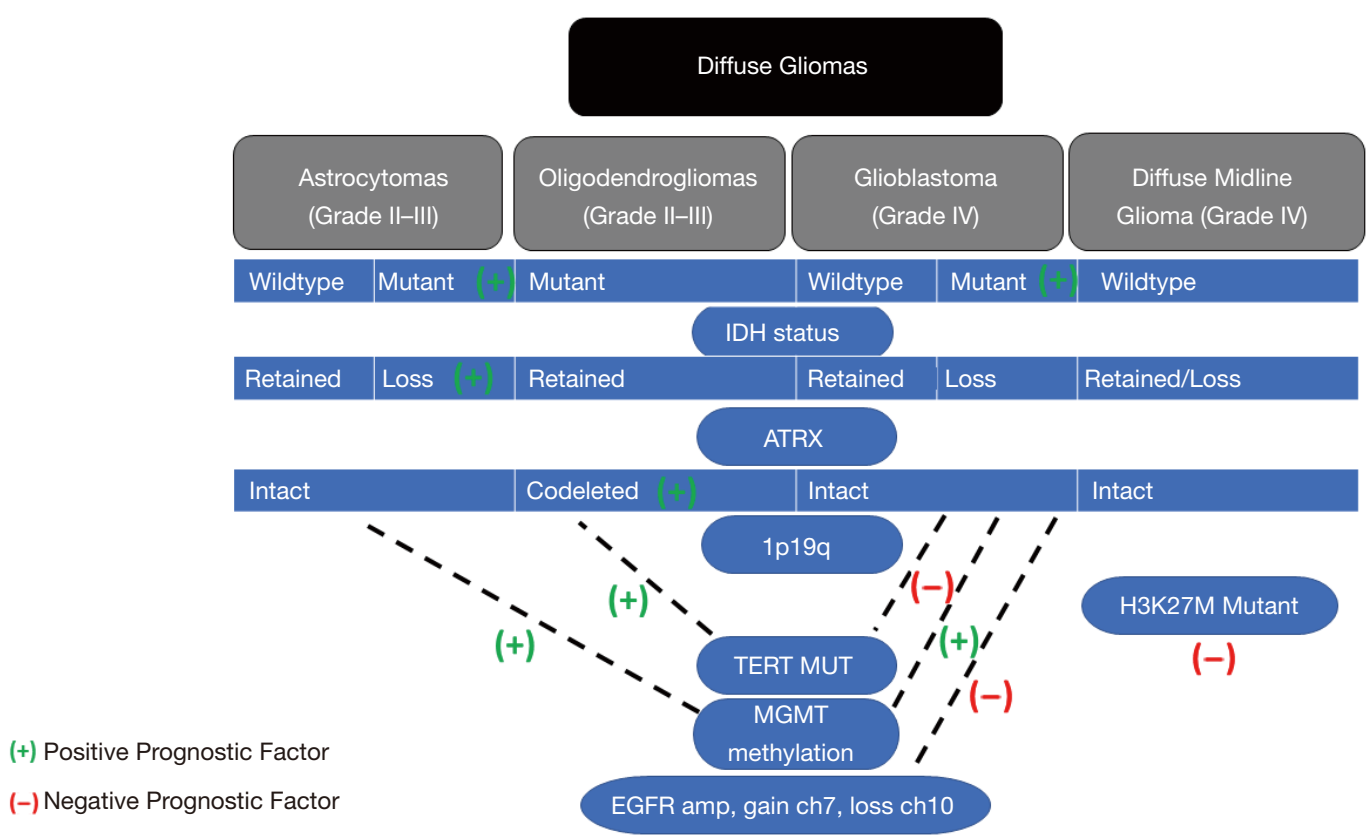

Figure 1 Molecular classification of diffuse gliomas.

identifiable systemic cause of death (pneumonia, sepsis, pulmonary embolus, etc.), while $7 \%$ had no identifiable cause of death even at autopsy (2). Of note, perioperative complications like hemorrhage, systemic emboli, infection and uncontrolled cerebral edema can occur before a formal diagnosis has even been made. It is increasingly recognized that all epilepsy patients are at risk of sudden death from seizures (Sudden Unexplained Death in Epilepsy Patients, or SUDEP), and brain tumor patients are likely not an exception. Studies in SUDEP have found that more frequent generalized seizures and sleeping alone are potentially modifiable risk factors (3). Many of these potentially fatal complications are stochastic in nature, and may occur at any time from initial diagnosis to end stage, increasing the unpredictable nature of prognostication at the individual patient level. Signs of progression that are present in other systemic malignancies such as metastatic disease or rising tumor markers are not present in gliomas. Functional status may remain very stable for quite some time before dropping precipitously, and is not always a reliable sign of disease progression; there is not the gradual, predictive, stepwise decline. Cognitive and neurological deficits may also be much more subtle and harder to easily identify as signs of progression. Prognostication in gliomas may therefore be considered to be much more challenging than with other malignancies (where it is already complex).

\section{Low grade glioma (LGGs)}

LGGs are classified as WHO grade II and are generally slow growing, infiltrative tumors presenting most commonly in the second to fourth decade of life (4). These can be identified incidentally on imaging in asymptomatic patients or may present with a broad range of symptoms depending on the location of the tumor. Symptoms may range from seizures, headaches, personality changes, cognitive deficits, to focal weakness or language deficits. Treatment is currently quite variable across centers around the world, in large part because systemic evidence remains limited. Randomized controlled trials in this field are challenging due to the rare nature of the tumor, slow growth, and variable outcomes.

The primary modality of treatment is surgical resection. While observation until clinical change and progression has been generally favored in most "low-risk" situationsconsidered to be younger (<40), asymptomatic patients with relatively small lesions-there is growing data to suggest that early resection may be safer and could possibly improve long term survival outcomes in all patients $(5,6)$. After a gross total or subtotal resection, the question of observation versus treatment with radiation and/or chemotherapy returns. Here, again, practice and recommendations can vary widely. There does exist evidence demonstrating 
that higher risk patients ( $>40$ or with subtotal resections) benefit from radiation and chemotherapy both in terms of progression-free survival (PFS) and overall survival (OS) (7). The type of chemotherapy used can vary from center to center [temozolomide versus the three-drug regimen procarbazine, lomustine and vincristine (PCV)]. In other centers, radiation can be delayed and the patient may be started on chemotherapy alone, given concerns of long-term impacts of radiation (especially in younger patients who might have several recurrences). Thus, the management of LGGs is a complicated, controversial area, with many facets that add to difficulties in prognostication for this patient population.

\section{Survival and prognostication}

Survival for LGG patients is quoted anywhere from 3 to 15 years, but this can be variable and dependent on a variety of factors that have been explored over the decades (Table 1) $(8,9)$. In most randomized studies, the 5 -year overall survival ranges from 58 to $72 \%$ (10). Older age—at least 40 years or above-portends a higher risk for progression of disease as discussed above, and places the patient in a category where resection or treatment should automatically be considered (11). Other factors that have similarly demonstrated a 'higher risk' for progression and poorer outcomes in large analyses have included astrocytic tumor type (instead of oligodendroglial type), tumor size $>6 \mathrm{~cm}$, tumor that crosses the midline, and neurological deficits prior to surgery $(11,12)$. Increased number of prognostic factors were associated with a shorter median overall survival (OS) (3.2 years with 3-5 factors, for instance) (11). Smaller studies have demonstrated significance of some other tumor specific factors which should be considered together with the more formal prognostic scores (13). The presence of contrast enhancement and rapid change over time has been associated with poorer outcomes (14-16). A poorer functional status from multimorbidity or neurological deficits also portends poorer prognosis, as do cognitive deficits $(17,18)$. Notably, epileptic seizures at diagnosis actually predicts a better prognosis and longer survival - this has been seen in many studies $(11,19)$. Recent data has also looked at the impact of race and ethnicity on survival, and found that both the incidence and survival rates differ by race. Non-Hispanic whites have a higher incidence of glioma, and their survival rate is lower when compared to Hispanic whites, blacks, Asians, and Pacific Islanders (20). This is true for all glioma types, whether low or high grade.
Molecular features have been linked to prognosis and survival in LGG and are increasingly being understood to trump other prognostic features (Figure 1). Most notably, an isocitrate dehydrogenase (IDH) mutation has been noted to be significantly correlated to positive prognosis in LGG and also suggests a higher rate of response to temozolomide (21). The same is true of the $1 \mathrm{p} 19 \mathrm{q}$ codeletion - the presence of this codeletion, which results in a diagnosis of oligodendroglioma, predicts an overall positive (PFS) and OS (22). The codeletion alone is a predictor of prolonged survival, even after accounting for other factors such as tumor grade, patient's age, size, etc. (23). The combination of a LGG that has both an IDH mutation and a $1 \mathrm{p} 19 \mathrm{q}$ codeletion can result in a 62 -month median PFS, compared to 48 months with the IDH mutation alone and 20 months for the IDH wildtype group (22). Recently, it has been shown that other molecular features including CDNK2A, CDK4 and chromosome 14 alterations are also more reliable than subjective tumor grade to predict prognosis (24) (Figure 1).

Additional mutations in the alpha thalassemia/ mental retardation syndrome X linked (ATRX) gene and Telomerase Reverse Transcriptase (TERT) promoter are also key and are gaining additional prominence as they have been better understood. TERT is involved in telomerase encoding, and ATRX plays a role in telomere maintenance. Mutations in either are mutually exclusive in gliomas. In lower grade gliomas, with $1 \mathrm{p} 19 \mathrm{q}$ codeletion and IDH mutant status, TERT mutations can be a positive prognostic factor (25). Loss of ATRX in IDH mutated astrocytomas (where there is no $1 \mathrm{p} 19 \mathrm{q}$ codeletion) is a positive prognostic factor, associated with improved PFS and OS (26) (Figure 1).

It can thus be synthesized from this evidence that the absolute worst prognosis in terms of PFS and OS can be attributed to the older ( $>40)$, highly symptomatic patient presenting with a large $(>5 \mathrm{~cm})$ astrocytoma, IDH wildtype, crossing the midline, which cannot be easily resected. We recognize that this particular patient may have a poor outcome, especially if they are already doing poorly prior to surgery with hemiplegia or seizures, or progress soon after initial resection. We also know that the higher risk patients should get treatment sooner at progression, and the treatment helps with their PFS and OS-but what treatment they get is variable, as discussed above $(7,22)$. However, the cases that are more challenging and harder to prognosticate are the patients that do not fall in the "worst prognosis" category, and either have a "good" or "mixed" prognosis. Examples might be the young patient with the 
Table 1 Positive and negative prognostic factors for diffuse gliomas

\begin{tabular}{|c|c|c|}
\hline Glioma type & Positive prognostic factor & Negative prognostic factor \\
\hline High grade glioma & $\begin{array}{l}\text { - Maximal safe resection; } \\
\text { - Age } 40 \text { or below; } \\
\text { - KPS } \geq 70 ; \\
\text { - IDH mutant status; } \\
\text { - MGMT methylation; } \\
\text { - 1p19q codeletion }\end{array}$ & $\begin{array}{l}\text { - Subtotal resection; } \\
\text { - Age >65; } \\
\text { - KPS } \leq 60 \text {; } \\
\text { - Non-Hispanic white race; } \\
\text { - Astrocytic lineage; } \\
\text { - Tumor size }>5 \mathrm{~cm} ; \\
\text { - Tumor crossing midline; } \\
\text { - Thalamic/brainstem locations; } \\
\text { - Neurological deficits at presentation; } \\
\text { - IDH wildtype status; } \\
\text { - TERT mutations (even IDH mutant status); } \\
\text { - EGFR amplification with gain of ch7, loss of ch10 (leads } \\
\text { to diagnosis of glioblastoma) }\end{array}$ \\
\hline
\end{tabular}

oligodendroglioma who has a very large tumor and is highly symptomatic, or an older patient with a small astrocytoma in an eloquent area causing significant symptoms. These patients, more typical of the population seen in clinic, do not follow the path identified in studies and their journeys are much harder to predict. In addition, the data above can generally only be applied to cases in the upfront setting, and cannot be easily translated to cases with recurrence or where there is progression after upfront resection or after initial radiation and chemotherapy.

\section{High grade glioma}

WHO Grade III and IV glioma comprise the "high grade glioma" (HGG) category. These rapidly progressing tumors are highly infiltrative and disabling, and their prognosis can be more limited than the lower grade gliomas, depending on a number of molecular features that are discussed below (27). Despite a large number of research trials through the decades, we remain quite limited in our ability to dramatically change the survival outcomes of these patients. Survival for grade IV astrocytoma, for example, remains in the range of $16-22$ months at this time.
Glioblastoma (GBM), the most common malignant brain tumor in adults, is a WHO grade IV astrocytoma. As with the other gliomas, there is no cure for GBM, and it remains a highly aggressive, malignant tumor. Whenever possible, a clinical trial should be a part of the patients' upfront treatment plan. Maximal safe resection has been recommended as the standard of care for essentially all gliomas, and this includes glioblastoma. This can improve outcomes when compared to a subtotal resection or biopsy alone (28). This may not be possible if the tumor is in an eloquent location, and morbidity of the surgery has to be constantly balanced with the benefit gained from the resection, since the patient still has to be functional postsurgery to undergo treatment.

Radiation and chemotherapy are the next steps in the treatment paradigm. Radiation should be initiated as soon as it is safe from a surgical wound perspective, ideally within 2-4 weeks. For patients younger than 65 with Karnofsky performance status (KPS) greater or equal to 60, optimal dose fractionation for external beam radiation therapy after resection or biopsy is 60 Gy delivered over 6 weeks. This schedule has demonstrated maximal benefit (Stupp) (29). In the elderly (>65) and those with a KPS 50 or above, 218$$
\text { . }
$$ 
hypofractionated radiotherapy has demonstrated similar survival with lesser side effects, at a dosing of $40 \mathrm{~Gy}$ in 15 fractions over 3 weeks (30). Those with KPS below 50 may attempt to get hypofractionated radiotherapy, one week of radiotherapy, chemotherapy alone, or best supportive care. Chemotherapy for both grade III and IV astrocytomas outside of a clinical trial may often starts with temozolomide, an oral methylating agent that is given concurrently with radiotherapy. This is followed by six monthly cycles of adjuvant temozolomide. This protocol demonstrated an improvement in median survival by 2.5 months (29). Notably, the benefits of the chemotherapy was most notable in the patient who had a methylation in the MGMT promoter gene in their tumors (31). Anaplastic oligodendrogliomas are rarer, but do occur, and have a generally better prognosis overall due to the $1 \mathrm{p} 19 \mathrm{q}$ codeletion discussed previously. PCV is used in several centers as the chemotherapy combination of choice in these cases, given a higher level of evidence, though randomized controlled trial data comparing outcomes against temozolomide is not yet available (32).

The NovoTTF-100A system known as Optune was approved by the FDA for patients with recurrent and newly diagnosed glioblastoma. This portable, non-invasive device generates low intensity, intermittent frequency alternating electric fields that are delivered to the patient's scalp via transducer arrays. These "tumor-treating fields," have anti-mitotic effects that interfere with mitotic spindle cell formation and chromosomal segregation during tumor cell division. Users must wear the device an average of 18 hours a day for best outcomes, and treatment starts with adjuvant temozolomide. Median survival improves to 20.5 months from 15.6 months with chemotherapy alone (33).

Recurrent disease-which eventually occurs in virtually all malignant gliomas-has limited options for treatment. There are a wide number of choices depending on the center, including anti-angiogenic therapy, re-operation, reradiation, clinical trials, etc.

\section{Survival and prognosis}

At this point we do know that patients with high grade glioma who tend to live longer are younger (in their 40s), have a high functional status at time of diagnosis (at least KPS 70), and are able to have a maximal resection (Table 1) $(34,35)$. As with LGG, large tumors that cross the midline have a worse prognosis than those that do not, as do deep thalamic or brainstem/cerebellar tumors (35). Elderly patients ( $>65$ ), with KPS $>80$, who have received a gross total resection do better than those who have a biopsy or no resection at all (36). In elderly patients, even a short course of adjuvant treatment can be better than no treatment at all, and thus the performance status should be considered over the actual age of the patient. A patient doing well postoperatively could get through hypofractionated radiation and chemotherapy and live up to 6 months longer than a patient who goes on hospice soon after diagnosis (37).

Figure 1 illustrates the molecular features that are important for glioblastomas, and can impact prognosis positively and negatively. IDH mutation status carries a strong prognostic value in glioblastoma - median survival of patients with IDH-mutant glioblastoma (which is a much smaller percentage of the overall number of cases) is much higher than that of IDH-wildtype glioblastoma. MGMT methylation is the second important factor. Methylation of the MGMT gene results in increased sensitivity to the chemotherapy agent temozolomide and thus patients with MGMT promoter methylation have been noted to have a median survival of 22 months on simply standard of care compared to 15 months for unmethylated patients (31). Combination of the IDH mutation and the MGMT methylation can further increase survival outcomes (38). TERT mutations have been associated with shorter survival when the mutation is associated with higher grade and IDH-mutant status (in contrast to their positive impact in lower grade gliomas) (25). MGMT methylated tumors, on the other hand, benefit from TERT mutation-it appears to increase therapeutic response to temozolomide in these cases (39).

In recent years, other molecular features have been identified that have changed the field and overall, there is a movement towards a more "molecular diagnosis" for glioblastoma based on these identifiers. Histologically low-grade appearing tumors can have these molecular features and behave like an aggressive, higher-grade tumor or a glioblastoma, and thus there is a growing argument that these should be treated as such upfront for the best outcomes. In fact, the benefit of these molecular features has been seen independent of other factors, as is the case with the loss of $1 \mathrm{p} 19 \mathrm{q}$. Molecular features that lead to a diagnosis of glioblastoma include the combination of EGFR amplification, gain of chromosome 7 with loss of chromosome 10. These alterations have been observed in the most aggressive form of glioblastoma-the IDHwildtype form, and there are several centers now that will upgrade any tumor that has these mutations on sequencing 
to a "molecular glioblastoma" diagnosis for treatment and prognostication purposes (40) (Figure 1). In addition, genome wide methylation patterns and copy number profiling have been shown to provide more accurate prognostic information (41).

Glioblastoma is itself adept in impacting the microenvironment and causing $\mathrm{T}$ cell dysfunction, impacting the immune system even prior to the start of any treatment (42). On top of this, the immunosuppressive nature of glioblastoma treatments themselves have a further prognostic impact. Radiation, temozolomide and steroids are all immunosuppressive but standard in the treatment course of HGG. Studies have demonstrated that patients may have a severe reduction in their CD 4 counts during their treatment, which in turn can impact survival and result in early death (43).

For a long time, the "extreme survivors" have baffled researchers and clinicians in the field-these are generally defined as glioblastoma patients who live $>5$ years with their disease. There is a wide range of variability in the field, but recent survival rates at 5 years are estimated to be close to $10 \%$ (44). Patients who live longer are more likely to become extreme survivors, studies have found, with those having reached the 2.5 -year mark more likely to survive to 5 years $(45,46)$. Additional studies are trying to focus on these survivors, collecting their numbers across institutions to determine patterns and predictive factors (44).

\section{Diffuse midline glioma}

With the WHO 2016 updated guidelines, the diffuse midline gliomas were recategorized and joined the grade IV astrocytoma category. These tumors were previously called diffuse intrinsic pontine gliomas and were thought to mainly occur in the pediatric population only. It is now recognized that these diffuse midline gliomas harbor a mutation in the $\mathrm{H} 3 \mathrm{~F} 3 \mathrm{~A}$ gene and are therefore known as $\mathrm{H} 3 \mathrm{~K} 27 \mathrm{M}$ mutant tumors, and can occur in adults and children alike, mainly in the brainstem and the midline structures (thalami, etc.). These are extremely aggressive and difficult to treat given a complete resection is almost always impossible and radiation can be complex and carry higher risks, though that remains the primary treatment modality. Temozolomide has been tried in these patients but there is no strong evidence to show its efficacy in this particular diagnosis, and there is some data to suggest that a large percentage of these tumors may lack MGMT methylation and thus be resistant to temozolomide (47). Other trials have been essentially unsuccessful until the last 2 years, but ONC201, an oral agent that is a dopamine receptor (D2/3) antagonist, was found to have some clinical benefit in small clinical trials and has since been expanded to larger trials for new and recurrent disease (48). Data has so far been very promising but remains in trial phase.

\section{Survival and prognosis}

Even with radiation, H3K27M mutated diffuse midline glioma has a median survival of 9 to 13 months, which is dismal even compared to glioblastoma (49). Without radiation this can be as little as 6 months. As discussed above, no chemotherapy exists that extends the survival significantly at this time, and tumor treating fields have not been tested in this group of patients (and are generally expected to be more successful in supratentorial and superficial tumors).

Data from the clinical trials for ONC201 is still early, but it seems that the drug is well tolerated with a good safety profile. The current estimate presented at the Society of Neuro-Oncology conference in 2019 for median progression free survival was 21.6 months for non-recurrent disease (in a very small group of patients), which is exciting news for this tumor that has had limited treatment options until this point (50).

\section{Overall recommendations regarding prognostication of glioma}

It is important to note that studies in the field for brain tumor prognostication generally note "median" survival numbers; the patients included in these trials usually fall on a range or a curve on either side of this median. Thus, there are often outliers who may do extremely poorly or may be "extreme survivors", for reasons that are still not completely clear to us. Thus, two patients at the same stage of tumor and treatment may demonstrate a great deal of variability. We all know that this is not unique to glioma and is seen in other cancers. The factual information we do have (as discussed above) can only provide a limited guidance in our estimates-but we remain very poor in our ability to prognosticate individual patient outcomes, even in the terminally ill (Christakis 2000).

Glioma patients and their loved ones will tend to have a very individual experience depending on their own particular tumor and its impact on their brain and behavior. Our goal in treating these tumors is always to 
extend quantity of life-extend the survival—without compromising significantly on quality of life. The care of every patient starts off with the shared hope that they will do as well as possible for as long as possible. Over time, however, it becomes important to reassess the clinical situation at regular periods and assess if clinical or tumor progression has limited our options enough to drastically change our expected prognosis and course.

\section{Clinical features of poor prognosis}

Clinical progression in brain tumors depends greatly on the location of the tumor. A small tumor in the brainstem may have a much higher burden of symptoms compared to a large tumor in the frontal hemisphere. Tumors in eloquent areas (motor strip, language processing zones) may be significantly disruptive from the very beginning, even if they are slow growing. Humans are resilient, however, and the stroke population has demonstrated to us the brain's significant ability to rehabilitate and heal. Brain tumor patients can similarly heal and recover from injury and surgery, but recurrence and progression can often present with return of or worsening of their symptoms. The neurological exam is therefore very important to follow. Early changes may be identified by the patients, their loved ones, or even the clinician, depending on the nature of symptoms. Patients may note new or worsening weakness, clumsiness, gait imbalance, or difficulty finding words or thinking. Breakthrough seizures may occur. Caregivers may note behavioral changes and new cognitive issues. Clinicians may note subtle new neurological deficits on exam corresponding to changes on imaging.

However, how heavily these changes weigh into the overall prognosis for the patient is dependent very much on where in the course the symptoms occur and how reversible they are. Every decline is evaluated against the background of the overall trajectory of the patient and their lines of treatment, steroid doses, etc., all must be taken into consideration. Having one or more of the signs of progression below, however, should raise concern and encourage the provider to re-evaluate plans of care. At this point, the presence of these symptoms does not appear to have been correlated with any specific time period of survival.

\section{Focal weakness}

The acute development of or subacute worsening of a focal deficit in glioma patients can contribute to their overall decline, especially if it does not reverse with steroids, antiangiogenic, therapy, or surgical treatment. These can leave them limited and dependent, bound to their bed/ wheelchair, and at increased risk for infection, thrombosis and ulcers. The change in ability, especially if dramatic, can also impact the patient's own hope and strength in these complex situations.

\section{Thrombosis}

Malignant brain tumors have some of the highest incidence of venous thrombosis, even in mobile patients, often leading to the use of anticoagulants which increases risk of bleeding complications (51). Caregivers should have a low threshold to screen for deep venous thrombosis, but in the palliative phase of care, the risks of anticoagulants should be carefully weighed against their benefits.

\section{Language disturbance}

As with weakness, impact on language can be profound, especially if it affects the patient's ability to express themselves or understand the world around them. Care can become extremely hard in these cases, and patients may again feel very vulnerable and hopeless.

\section{Seizures}

While seizures at presentation for LGG are actually a positive prognostic sign, breakthrough seizures can also be a sign of progression and recurrence for gliomas and especially glioblastomas (52). New onset generalized or partial status epilepticus is another concerning sign that suggests worsening cerebral dysfunction. Seizures can be alarming for both patients and caregivers and contribute to significant anxiety, but should be considered preventable at all stages. Rarely, seizures themselves can be the cause of death and some of this risk can be mitigated by antiepileptic drugs improving control of generalized seizures, and possibly by the presence of caregivers overnight (3). In later stages of disease when swallowing is impaired, agents with alternate access routes may need to be considered.

\section{Mood disturbance and personality changes}

These symptoms can often be subtle and may be picked up on by caregivers before the patient notes them, and brought to the attention of the clinician. They may range 
from anxiety and mild depression to significant frontal disinhibition and even psychosis (though this is rare). New presentations of these symptoms can be concerning for tumor progression but can also result in overall emotional and physical decline, and can impact prognosis. Though suicidal ideation and depression are common in patients with brain tumors, suicide as a cause of death seems quite rare, though not rigorously studied (53) (see accompanying article in this edition by Gibson $e t a l$.).

\section{Cognitive decline}

Cognitive decline in glioma patients is multifactorial and likely underreported-the tumor, radiation, chemotherapy, steroids, anti-epileptics, all contribute to the cognitive decline which becomes more notable as the patient deteriorates. Concurrent medications, especially corticosteroids and anti-epileptics should always be considered as potentially correctable causes of cognitive and mood changes. Insomnia is a very common side effect of corticosteroids and can contribute to cognitive impairment. Patients are noted to be increasingly dependent on caregivers for independent activities of daily living and gradually for activities of daily living. This is overall a very concerning sign, depending on the pre-existing functional status.

\section{Fatigue}

This is a significant symptom for brain tumor patients from the very beginning, but this tends to get worse as the disease progresses and towards the end of life. Again, this is multifactorial. Glioma, radiation, chemotherapy, and medications all contribute to fatigue. There are also emotional and mood contributors to this condition. Patients and caregivers will note increased hours of sleep, and more naps throughout the day. There will be less energy for activities and events with family members. Corticosteroids and some anti-epileptics can impair normal sleep, and should be assessed as a potentially treatable cause. Stimulants such as methylphenidate and modafinil have been studied in this population with no significant proven benefit at this point.

\section{Dysphagia}

Swallowing difficulties may present early with brainstem or motor cortex tumors, but can present with all brain tumors and can have a significant impact on the conversations surrounding prognosis. Depending on the severity of the swallowing difficulties, patients may have low enough oral intake to develop malnutrition and are at risk of aspiration pneumonia. Neurologic cause of the dysphagia is likely progressive and irreversible at this point and artificial feeding will not change outcomes.

\section{Steroid dependence}

Dexamethasone is often dosed with LGG and HGG to treat a wide range of symptoms. Often, patients are placed on a quick taper and see rapid benefit and are able to wean off without significant return of symptoms. Steroid dependence-the inability to wean off steroids or even taper down steroid dosages-especially earlier in the course of the glioma is a proven negative prognostic factor $(54,55)$. Patients are often placed on steroids towards the last stages of their life and remain on them until the end of life, but the symptomatic benefits of corticosteroids (reducing neurologic symptoms) should always be weighed against their many negative effects (heartburn, insomnia, mood changes, weight gain, infections, etc.).

\section{Prognostic understanding of patients and caregivers}

Research specifically on how patients with glioma and their caregivers understand their prognosis is limited at this time. The literature that does exist demonstrates patients and caregivers believe in the importance of the prognostic information $(56,57)$. Memory impairment in glioma patients may make understanding this prognosis especially challenging, and it does seem that patients do not understand their life expectancy, especially if they have proven impairment (56). Caregivers, on the other hand, do appear to have awareness of the incurability of the disease and possess more accurate understanding of the survival estimate (57). It should be communicated to patients and families that prognostic information may be accurate at the population level, but individual patient predictions are not at all accurate, even by experienced specialists. In one prospective study of patients being referred to hospice services, only $40 \%$ of patients died within 1 month of their doctors' predictions (58). The stochastic nature of some causes of death like seizures, infections and thrombosis likely increases this inaccuracy significantly (2). This prognostic discord can be very significant and may have 
implications at the end of life in terms of the distress it may cause as well as conflict in shared decision making. A small prospective pilot study of HGG patients and their caregivers noted that prognostic understanding fluctuated every month during adjuvant treatment, and varied widely from each other and from their providers (which remained quite static) (59).

The timing of communication of this prognostic information is important-Lobb et al. has found that initial communication of the diagnosis is a time when patients and caregivers are consumed by shock and simply processing the information, and focused on preserving hope (60). Hope is crucial for the brain tumor patient and the brain will protect the mind from reality by creating hope in this condition (61). The communication of prognosis in glioma has to weigh the delicate balance of preserving hope while constantly practicing honest communication at the right time, as to prepare the patient and their loved ones for the future ahead $(62,63)$. It is important to note that conversations on prognosis should not be single, static conversations-as reviewed exhaustively above, prognostication in glioma is an uncertain science- the prognosis may be dynamic, and ever-changing (63). Communication should be honest with room for uncertainty and shared optimism.

\section{Summary and conclusions}

Patients and caregivers should be aware that information on prognosis is not highly accurate at the individual patient level and that unpredictable life-threatening complications can occur even in the early phase of death. Some of these complications such as seizures, injuries, infections, aspiration and thrombosis may be at least partially preventable or treatable. While certain markers for positive and negative prognosis exist, it is difficult to truly predict an individual journey. Instead, providers must aim to constantly re-evaluate the patient condition, and re-evaluate their own assessment of prognosis, sharing this with the patient and caregiver when appropriate. Communications about prognosis should be honest but allow for uncertainty, and acknowledge the challenge of providing firm guidance. There can always be room for hope, even while preparing for the worst stages of this disease.

\section{Acknowledgments}

Funding: None.

\section{Footnote}

Provenance and Peer Review: This article was commissioned by the editorial office, Annals of Palliative Medicine for the series "Palliative Care in Neuro-Oncology". The article has undergone external peer review.

Peer Review File: Available at http://dx.doi.org/10.21037/ apm-20-640

Conflicts of Interest: Both authors have completed the ICMJE uniform disclosure form (available at http://dx.doi. org/10.21037/apm-20-640). The series "Palliative Care in Neuro-Oncology" was commissioned by the editorial office without any funding or sponsorship. JJG served as the unpaid Guest Editor of the series. Both authors have no other conflicts of interest to declare.

Ethical Statement: The authors are accountable for all aspects of the work in ensuring that questions related to the accuracy or integrity of any part of the work are appropriately investigated and resolved.

Open Access Statement: This is an Open Access article distributed in accordance with the Creative Commons Attribution-NonCommercial-NoDerivs 4.0 International License (CC BY-NC-ND 4.0), which permits the noncommercial replication and distribution of the article with the strict proviso that no changes or edits are made and the original work is properly cited (including links to both the formal publication through the relevant DOI and the license). See: https://creativecommons.org/licenses/by-nc-nd/4.0/.

\section{References}

1. Louis DN, Perry A, Reifenberger G, et al. The 2016 World Health Organization Classification of Tumors of the Central Nervous System: a summary. Acta Neuropathol 2016;131:803-20.

2. Silbergeld DL, Rostomily RC, Alvord EC. The cause of death in patients with glioblastoma is multifactorial: clinical factors and autopsy findings in 117 cases of supratentorial glioblastoma in adults. J Neurooncol 1991;10:179-85.

3. Sveinsson $\mathrm{O}$, Andersson T, Mattsson $\mathrm{P}$, et al. Clinical risk factors in SUDEP: A nationwide population-based casecontrol study. Neurology 2020;94:e419-29.

4. Ostrom QT, Gittleman H, Truitt G, et al. CBTRUS 
Statistical Report: Primary Brain and Other Central Nervous System Tumors Diagnosed in the United States in 2011-2015. Neuro Oncol 2018;20:iv1-86.

5. Jakola AS, Skjulsvik AJ, Myrmel KS, et al. Surgical resection versus watchful waiting in low-grade gliomas. Ann Oncol 2017;28:1942-8.

6. Aghi MK, Nahed BV, Sloan AE, et al. The role of surgery in the management of patients with diffuse low grade glioma: A systematic review and evidence-based clinical practice guideline. J Neurooncol 2015;125:503-30.

7. Buckner JC, Shaw EG, Pugh SL, et al. Radiation plus Procarbazine, CCNU, and Vincristine in Low-Grade Glioma. N Engl J Med 2016;374:1344-55.

8. Hottinger AF, Hegi ME, Baumert BG. Current management of low-grade gliomas. Curr Opin Neurol 2016;29:782-8.

9. Claus EB, Black PM. Survival rates and patterns of care for patients diagnosed with supratentorial low-grade gliomas: data from the SEER program, 1973-2001. Cancer 2006;106:1358-63.

10. Soffietti R, Baumert BG, Bello L, et al. Guidelines on management of low-grade gliomas: report of an EFNSEANO Task Force. Eur J Neurol 2010;17:1124-33.

11. Pignatti F, van den Bent M, Curran D, et al. Prognostic factors for survival in adult patients with cerebral lowgrade glioma. J Clin Oncol 2002;20:2076-84.

12. Gorlia $T, W u W$, Wang $M$, et al. New validated prognostic models and prognostic calculators in patients with lowgrade gliomas diagnosed by central pathology review: a pooled analysis of EORTC/RTOG/NCCTG phase III clinical trials. Neuro Oncol 2013;15:1568-79.

13. Smits A, Jakola AS. Clinical Presentation, Natural History, and Prognosis of Diffuse Low-Grade Gliomas. Neurosurg Clin N Am. 2019;30(1):35-42.

14. Pallud J, Capelle L, Taillandier L, et al. Prognostic significance of imaging contrast enhancement for $\mathrm{WHO}$ grade II gliomas. Neuro Oncol 2009;11:176-82.

15. Pallud J, Blonski M, Mandonnet E, et al. Velocity of tumor spontaneous expansion predicts long-term outcomes for diffuse low-grade gliomas. Neuro Oncol 2013;15:595-606.

16. Waqar M, Hanif S, Brodbelt AR, et al. Prognostic Factors in Lobar World Health Organization Grade II Astrocytomas. World Neurosurg 2015;84:154-62.

17. Chang EF, Smith JS, Chang SM, et al. Preoperative prognostic classification system for hemispheric low-grade gliomas in adults. J Neurosurg 2008;109:817-24.

18. Daniels TB, Brown PD, Felten SJ, et al. Validation of EORTC prognostic factors for adults with low-grade glioma: a report using intergroup 86-72-51. Int J Radiat Oncol Biol Phys 2011;81:218-24.

19. Pallud J, Audureau E, Blonski M, et al. Epileptic seizures in diffuse low-grade gliomas in adults. Brain 2014;137:449-62.

20. Ostrom QT, Cote DJ, Ascha M, et al. Adult Glioma Incidence and Survival by Race or Ethnicity in the United States From 2000 to 2014. JAMA Oncol 2018;4:1254-62.

21. Houillier C, Wang X, Kaloshi G, et al. IDH1 or IDH2 mutations predict longer survival and response to temozolomide in low-grade gliomas. Neurology 2010;75:1560-6.

22. Baumert BG, Hegi ME, van den Bent MJ, et al. Temozolomide chemotherapy versus radiotherapy in high-risk low-grade glioma (EORTC 22033-26033): a randomised, open-label, phase 3 intergroup study. Lancet Oncol 2016;17:1521-32.

23. Smith JS, Perry A, Borell TJ, et al. Alterations of chromosome arms $1 \mathrm{p}$ and $19 \mathrm{q}$ as predictors of survival in oligodendrogliomas, astrocytomas, and mixed oligoastrocytomas. J Clin Oncol 2000;18:636-45.

24. Cimino PJ, Holland EC. Targeted copy number analysis outperforms histologic grading in predicting patient survival for WHO grades II/III IDH-mutant astrocytomas. Neuro Oncol 2019;21:819-21.

25. Eckel-Passow JE, Lachance DH, Molinaro AM, et al. Glioma Groups Based on 1p/19q, IDH, and TERT Promoter Mutations in Tumors. N Engl J Med. 2015;372:2499-508.

26. Wiestler B, Capper D, Holland-Letz T, et al. ATRX loss refines the classification of anaplastic gliomas and identifies a subgroup of IDH mutant astrocytic tumors with better prognosis. Acta Neuropathol 2013;126:443-51.

27. Alexander BM, Cloughesy TF. Adult Glioblastoma. J Clin Oncol 2017;35:2402-9.

28. Trifiletti DM, Alonso C, Grover S, et al. Prognostic Implications of Extent of Resection in Glioblastoma: Analysis from a Large Database. World Neurosurg 2017;103:330-40.

29. Stupp R, Mason WP, van den Bent MJ, et al. Radiotherapy plus concomitant and adjuvant temozolomide for glioblastoma. N Engl J Med 2005;352:987-96.

30. Perry JR, Laperriere N, O'Callaghan CJ, et al. ShortCourse Radiation plus Temozolomide in Elderly Patients with Glioblastoma. N Engl J Med 2017;376:1027-37.

31. Hegi ME, Diserens AC, Gorlia T, et al. MGMT gene silencing and benefit from temozolomide in glioblastoma. N Engl J Med 2005;352:997-1003. 
32. Cairncross G, Wang M, Shaw E, et al. Phase III trial of chemoradiotherapy for anaplastic oligodendroglioma: long-term results of RTOG 9402. J Clin Oncol 2013;31:337-43.

33. Stupp R, Taillibert S, Kanner AA, et al. Maintenance Therapy With Tumor-Treating Fields Plus Temozolomide vs Temozolomide Alone for Glioblastoma: A Randomized Clinical Trial. JAMA 2015;314:2535-43.

34. Scott JN, Rewcastle NB, Brasher PM, et al. Which glioblastoma multiforme patient will become a longterm survivor? A population-based study. Ann Neurol 1999;46:183-8.

35. Chaudhry NS, Shah AH, Ferraro N, et al. Predictors of long-term survival in patients with glioblastoma multiforme: advancements from the last quarter century. Cancer Invest. 2013;31:287-308.

36. Heiland DH, Haaker G, Watzlawick R, et al. One decade of glioblastoma multiforme surgery in 342 elderly patients: what have we learned? J Neurooncol 2018;140:385-91.

37. Tsang DS, Khan L, Perry JR, et al. Survival outcomes in elderly patients with glioblastoma. Clin Oncol (R Coll Radiol) 2015;27:176-83.

38. Millward CP, Brodbelt AR, Haylock B, et al. The impact of MGMT methylation and IDH-1 mutation on long-term outcome for glioblastoma treated with chemoradiotherapy. Acta Neurochir (Wien) 2016;158:1943-53.

39. Arita H, Yamasaki K, Matsushita Y, et al. A combination of TERT promoter mutation and MGMT methylation status predicts clinically relevant subgroups of newly diagnosed glioblastomas. Acta Neuropathol Commun 2016;4:79.

40. Stichel D, Ebrahimi A, Reuss D, et al. Distribution of EGFR amplification, combined chromosome 7 gain and chromosome 10 loss, and TERT promoter mutation in brain tumors and their potential for the reclassification of IDHwt astrocytoma to glioblastoma. Acta Neuropathol 2018;136:793-803.

41. Cimino PJ, McFerrin L, Wirsching HG, et al. Copy number profiling across glioblastoma populations has implications for clinical trial design. Neuro Oncol 2018;20:1368-73.

42. Woroniecka KI, Rhodin KE, Chongsathidkiet $\mathrm{P}$, et al. T-cell Dysfunction in Glioblastoma: Applying a New Framework. Clin Cancer Res 2018;24:3792-802.

43. Grossman SA, Ye X, Lesser G, et al. Immunosuppression in patients with high-grade gliomas treated with radiation and temozolomide. Clin Cancer Res 2011;17:5473-80.

44. Johnston SK, Whitmire P, Massey SC, et al. ENvironmental Dynamics Underlying Responsive
Extreme Survivors (ENDURES) of Glioblastoma: A

Multidisciplinary Team-based, Multifactorial Analytical Approach. Am J Clin Oncol 2019;42:655-61.

45. Johnson DR, Ma DJ, Buckner JC, et al. Conditional probability of long-term survival in glioblastoma: a population-based analysis. Cancer 2012;118:5608-13.

46. Smoll NR, Schaller K, Gautschi OP. Long-term survival of patients with glioblastoma multiforme (GBM). J Clin Neurosci 2013;20:670-5.

47. Abe H, Natsumeda M, Okada M, et al. MGMT Expression Contributes to Temozolomide Resistance in H3K27M-Mutant Diffuse Midline Gliomas. Front Oncol 2020;9:1568.

48. Chi AS, Tarapore RS, Hall MD, et al. Pediatric and adult H3 K27M-mutant diffuse midline glioma treated with the selective DRD2 antagonist ONC201. J Neurooncol 2019;145:97-105.

49. Pratt D, Natarajan SK, Banda A, et al. Circumscribed/nondiffuse histology confers a better prognosis in H3K27Mmutant gliomas. Acta Neuropathol 2018;135:299-301.

50. Rahman Kawakibi A, Gardner S, Chi A, et al. PDCT-12. Clinical efficacy of onc201 in thalamic H3 K27M-mutant glioma. Neuro-Oncology. 2019;21:vi186-vi.

51. Mantia C, Zwicker JI. Anticoagulation in the Setting of Primary and Metastatic Brain Tumors. Cancer Treat Res 2019;179:179-89.

52. Chaichana KL, Parker SL, Olivi A, et al. Long-term seizure outcomes in adult patients undergoing primary resection of malignant brain astrocytomas. Clinical article. J Neurosurg 2009;111:282-92.

53. Seddighi A, Seddighi AS, Nikouei A, et al. Psychological aspects in brain tumor patients: A prospective study. Hell J Nucl Med 2015;18 Suppl 1:63-7.

54. Lote K, Egeland T, Hager B , et al. Survival, prognostic factors, and therapeutic efficacy in low-grade glioma: a retrospective study in 379 patients. J Clin Oncol 1997;15:3129-40.

55. Hohwieler Schloss M, Freidberg SR, Heatley GJ, et al. Glucocorticoid dependency as a prognostic factor in radiotherapy for cerebral gliomas. Acta Oncol 1989;28:51-5

56. Diamond EL, Prigerson HG, Correa DC, et al. Prognostic awareness, prognostic communication, and cognitive function in patients with malignant glioma. Neuro Oncol 2017;19:1532-41.

57. Applebaum AJ, Buda K, Kryza-Lacombe M, et al. Prognostic awareness and communication preferences among caregivers of patients with malignant glioma. 
Psychooncology 2018;27:817-23.

58. Christakis NA, Lamont EB. Extent and determinants of error in doctors' prognoses in terminally ill patients: prospective cohort study. BMJ 2000;320:469-72.

59. Sharma A, Wolfe E, Barrera C, et al. QOLP-15. Longitudinal perception of prognosis of patients with high grade glioma compared to their caregivers and clinicians. Neuro-Oncology 2019;21:vi200-1.

60. Lobb EA, Halkett GK, Nowak AK. Patient and caregiver perceptions of communication of prognosis in high grade glioma. J Neurooncol 2011;104:315-22.

Cite this article as: Sharma A, Graber JJ. Overview of prognostic factors in adult gliomas. Ann Palliat Med 2021;10(1):863-874. doi: 10.21037/apm-20-640
61. Salander P, Bergenheim T, Henriksson R. The creation 888 of protection and hope in patients with malignant brain $\quad 889$ tumours. Soc Sci Med 1996;42:985-96.

62. Diamond EL, Corner GW, De Rosa A, et al. Prognostic awareness and communication of prognostic information in malignant glioma: a systematic review. J Neurooncol 2014;119:227-34.

63. Rosenblum ML, Kalkanis S, Goldberg W, et al. Odyssey of hope: a physician's guide to communicating with brain tumor patients across the continuum of care. J Neurooncol 2009;92:241-51. 